\title{
Proximal weakness of the extremities as main feature of amyloid myopathy
}

\author{
F G I JENNEKENS, J H J WOKKE
}

From the Laboratory of Neuromuscular Diseases, Department of Neurology, University Hospital, Utrecht, The Netherlands

SUMMARY Two patients with muscle weakness caused by amyloid myopathy are described. Characteristic features such as pseudohypertrophy and abnormal firmness, and tumours of muscles were absent. It is suggested that muscle weakness in amyloid myopathy is caused by layers of amyloid covering muscle fibres. In middle aged or elderly patients with proximal muscle weakness the diagnosis of amyloid myopathy should be considered.

The clinical picture of amyloid myopathy has attracted little interest in recent handbooks on myology or muscle pathology..$^{1-4}$ The clinical signs considered as hallmarks of this rare disorder are nevertheless of interest: pseudomuscular hypertrophy, palpable tumours within muscles, "wooden consistency" of skeletal muscles, macroglossia, hoarseness of the voice, loss of mobility and some degree of muscle weakness. $^{5-7}$

We describe two patients with weakness and muscle atrophy as the main characteristics of amyloid myopathy, and demonstrate that the clinical picture of amyloid myopathy may be more variable than is apparent from the literature. The ultrastructural studies of the three muscle biopsies from these patients provide a possible explanation for this variation.

\section{Case histories}

Case 1

A 68 year old female was admitted because of progressive weakness of the limbs. She had been in good health until the age of 65 years, when she started to complain of fatigue, constipation and weakness when cycling or mounting stairs. IgA lambda paraproteinaemia had been discovered and myeloma had been diagnosed when bone marrow puncture disclosed mild proliferation of plasma cells. When 67 she noticed inability to raise the arms above the head and some muscle stiffness. Her voice gradually became hoarse and she experienced difficulty in swallowing. At the same time a ductal carcinoma of the breast was diagnosed and removed.

Address for reprint requests: Prof Frans G I Jennekens, Laboratory of Neuromuscular Diseases, University Hospital, P.O. Box 16150, 3500 CG Utrecht, The Netherlands.

Received 14 October 1986. Accepted 11 February 1987
Examination after mastectomy revealed that she had a hoarse voice but no macroglossia, muscular hypertrophy or obvious atrophy. Abnormal firmness of the musculature was absent. There was weakness of neck flexors, grade 4 (MRC scale), shoulder girdle and thigh muscles, 3-4 and 4, and extensors of the feet, 4 . Tendon reflexes were present and sensation and coordination were undisturbed.

ESR was $33 \mathrm{~mm}$ after one hour. Routine haematological and urine examination were normal. Serum creatinine kinase activity $(\mathrm{CK})$ was $416 \mathrm{U} / 1$ (normal < 105). Serum immuno-electrophoresis showed IgA lambda paraproteins. Serum IgA was $19 \mathrm{~g} / 1$ (normal 0.5-2.6 g/1), IgM 0.3 g/l (normal 0.4-2.2 g/l), IgG $5 \cdot 1$ (normal 4.7-13.3 g/l). Bone marrow examination revealed a plasmacytosis of $15 \%$ (normal 0.5-3) with immature IgA lambda positive plasma cells. A Bence Jones test of the urine was negative. Radio-isotope total body scanning and radiographs of the skeleton were normal. An ECG showed premature ventricular contractions and negative left precordial T-spikes. Electromyography (EMG) showed fibrillations and positive sharp waves with an excess of polyphasic muscle action potentials of small amplitude and brief duration. Motor nerve conduction velocity was normal. Computed tomography of the musculature at eight levels demonstrated atrophy of thigh and calf muscles and slight hypertrophy of the triceps brachii muscles. Biopsies of the deltoid and vastus lateralis muscles revealed amyloid myopathy (vide infra). She was treated with alkeran and prednisone. Her condition worsened and muscular weakness increased but muscle hypertrophy, macroglossia and tumours within muscles did not develop. She died suddenly from cardiac arrest at the age of 69 years. Necropsy was refused.

\section{Case 2}

A 54 year old woman was admitted with complaints about bluish discolouration of the skin for 3 years, muscular weakness, fatigue and shortness of breath for 2 years, and for a few months a slightly painful and heavy sensation in shoulder- and upper arm muscles with inability to keep the arms raised above the head and difficulty in swallowing. 
On examination, slight rubbing of the skin was found to result in loosening of the epidermis from the underlayer and ecchymosis. The liver was enlarged. Voice and tongue were normal and the musculature was not unduly firm. The shoulder girdle muscles were atrophic. The neck flexors were mildly weak, MRC grade 4 . The patient had wing scapulae. There was weakness of the trapezius, rhomboideus, pectoralis and deltoid muscles, 3-4, of the biceps brachii, 4, triceps brachii, 4-5, and wrist extensors, 4-5, hipflexors, 3-4, hamstrings and extensors of the feet, 4 . Sensation was normal and tendon reflexes were low but elicitable.

Extensive laboratory examination revealed the following abnormalities: ESR $62 \mathrm{~mm}$ after one hour, acid phosphatase $14 \cdot 2$ U/1 (normal 4-11), CK 402 U/1. Serumprotein level was decreased $(53 \mathrm{~g} / 1$, normal values between 62 and 78$)$. Immuno-electrophoresis of serum showed low IgA (0.23 $\mathrm{g} / \mathrm{l}), \operatorname{IgG}(2 \cdot 4 \mathrm{~g} / \mathrm{l})$, and $\operatorname{IgM}(0 \cdot 16 \mathrm{~g} / \mathrm{l})$, and free lambda chains. There was a lambda type Bence Jones proteinuria. Bone marrow examination revealed a plasmacytosis of $38 \%$ (normal 0.5-3) with immature and abnormal plasma cells. Myeloma was diagnosed. Plasma cells were IgG lambda positive by immuno-fluorescence. Radiological examination of the skeleton disclosed no bone lesions. The ECG showed low voltages and was considered abnormal. Electromyography revealed fibrillation potentials with positive sharp waves and polyphasic motor unit potentials. Amyloid was found in biopsy specimens from the skin, kidney and skeletal muscles (vide infra). Treatment with alkeran and prednisone was started. In the following months renal func- tion deteriorated and bullous skin lesions developed on both legs. Muscle weakness was progressive, but hypertrophy did not develop. She died at the age of 55 years from cardiac arrythmia. Postmortem examination showed amyloid deposits in the vessel walls in kidney, spleen, adrenal cortex, and in cardiac and skeletal muscles.

\section{HISTOPATHOLOGY}

The changes in the muscle (two biopsies from case 1 and one from case 2) differed only in degree, and will therefore be described together. In transverse cryostat sections, the majority of the muscle fibres were normal in size and shape and did not show any structural abnormalities. There was disseminated atrophy of type I and II fibres, least in the first biopsy of case 1 (fig 1). In transverse sections, atrophic fibres were slightly elongated, round or oval and somewhat irregular in shape. Occasional fibres showed splitting, necrosis or regenerative features. Some small vessels and some atrophic fibres were surrounded by hyalin material. Congo red staining was positive and potassium permanganate resistant. ${ }^{8}$ The presence of amyloid deposits was easier to detect by fluorescence than by the birefrimgence of congo red stained sections (fig 1). ${ }^{8}$ Myosin ATP-ase activity revealed a mosaic pattern of type I and II fibres with occasional small type groups in the deltoid muscles of case 1.

Electron microscopy showed non-branching filaments which were approximately $9 \mathrm{~nm}$ in diameter in the endomysial space. Accumulations of such amyloid filaments occurred in a characteristic pattern. Many, though not all,

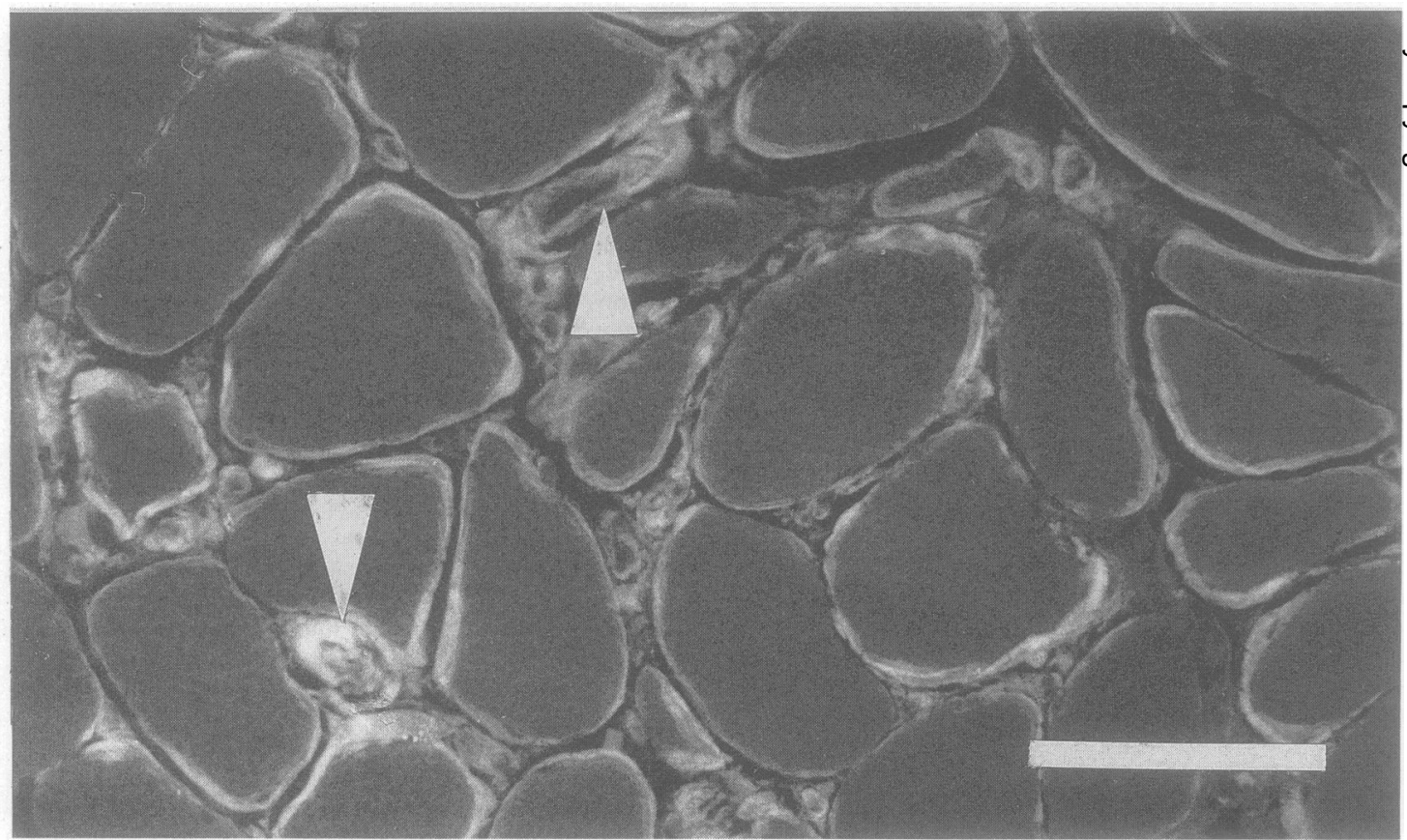

Fig 1 Fluorescing, congo red stained material in a transverse cryostat section (case 1, left deltoid muscle). Fluorescence is seen around most of the muscle fibres, around some small vessels (arrowheads) and at some other places in the endomysium. There is an abnormal variation in size of the muscle fibres. Bar: $1 \mu \mathrm{m}$. 


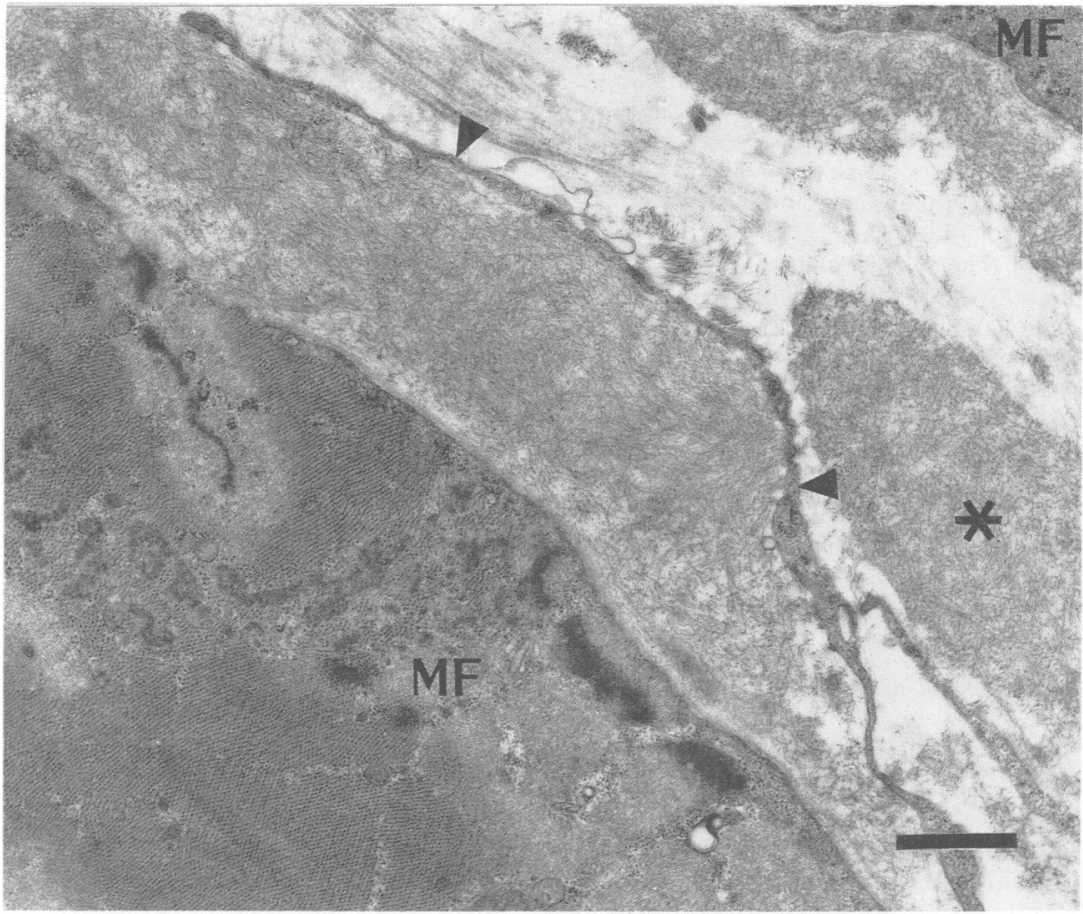

Fig 2 Electronmicrograph (case 1, left vastus lateralis muscle). Layers of closely packed filaments are covering the muscle fibres $(M F)$ at the lower left and the upper right. The layer covering the fibre at the lower left is bounded by an elongated fibroblast (arrowhead). Part of an oval mass of amyloid filaments is present on the right (asterisk) in the endomysial space.

Elsewhere, the endomysium contains collagen and elastic fibres but no filament. Bar: $1 \mu \mathrm{m}$.

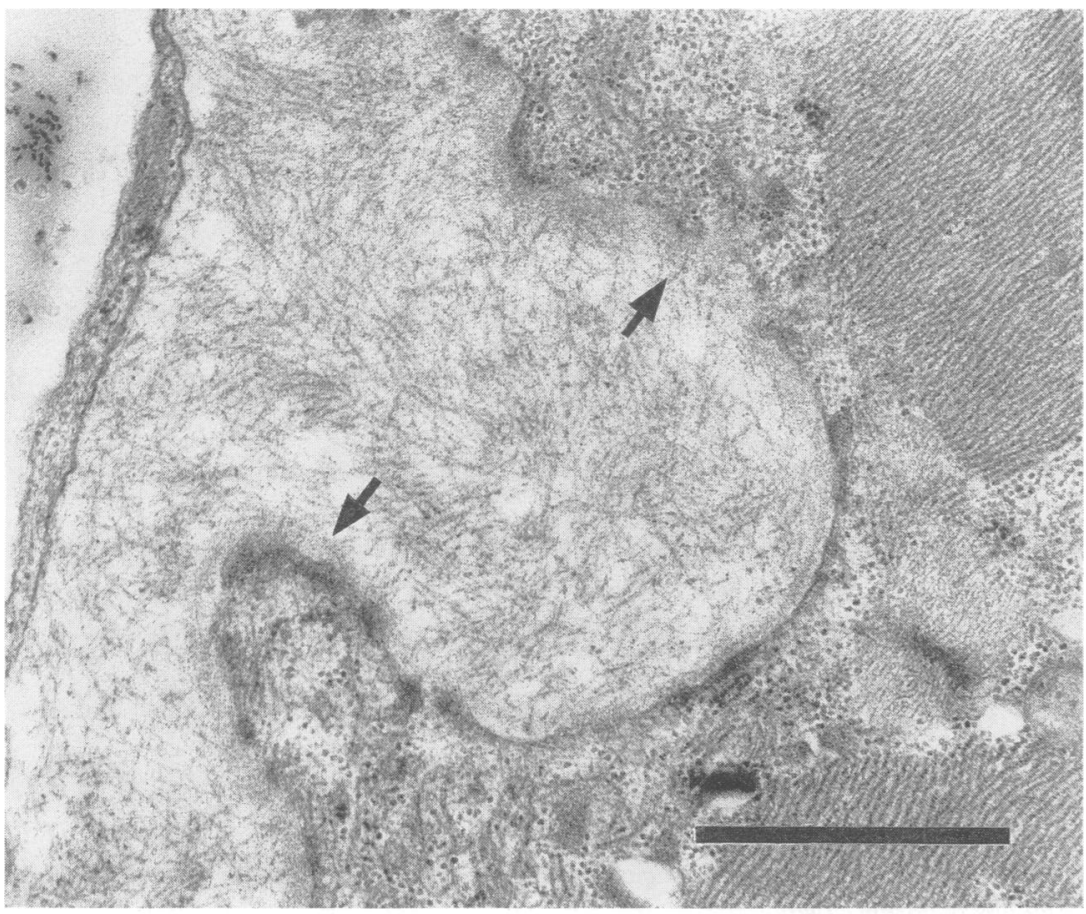

Fig 3 Electronmicrograph (same biopsy as fig 2). An accumulation of straight and unbranched filaments is present in between a muscle fibre and a fibroblast. Filaments do not pierce through the basal lamina but occasionally they do intrude into the lamina (arrows). Bar: $1 \mu \mathrm{m}$. 
muscle fibres were covered by layers of amyloid filaments of variable thickness (fig 2). In places, the layers were bounded by elongated fibroblasts. Amyloid filaments within these layers were distributed randomly. The layers closely joined the basal lamina, but did not pierce through the lamina (fig 3). No amyloid filaments were seen in muscle fibres. Some of the so-covered muscle fibres had developed capricious and elongated folds which occasionally followed the surface of the muscle fibre for some distance (fig 4). The folds contained various sarcoplasmic organels but no myofibrils. Folding of muscle fibres was seen more frequently and was more extensive in the biopsy of the second patient than in those of the first patient. Practically all small intramuscular vessels were surrounded by layers of amyloid filaments (fig 5). In places oval or round masses of amyloid filaments were seen which were partly bounded by elongated fibroblasts (fig 6). These masses were often located near small vessels (fig 5). Amyloid filaments were not randomly distributed in the endomysial spaces.

\section{Discussion}

Classification of the amyloidoses is based in part upon the chemistry of the amyloid fibrils. Amyloidosis accompanying plasma cell dyscrasia is characterised by fibrils containing a component of immunoglobulin light chains. In patients with chronic, often sup- purative disease, the amyloid fibrils contain so called AA (amyloid A) protein and in familial amyloid polyneuropathy part of the protein in the amyloid fibrils is related to serum prealbumin. ${ }^{9}$ It is not exceptional for amyloid to infiltrate striated musculature. In patients with plasma cell dyscrasia and amyloidosis, the heart musculature is frequently involved. ${ }^{10}$ The skeletal muscle is infiltrated as a rule in patients with amyloid polyneuropathy. ${ }^{11}$

The synthesis of amyloid filaments is at present poorly understood. Endothelial cells, histocytes, mesangial cells in the renal glomerulus and other cells may absorb amyloid precursor proteins from the blood, and convert these into amyloid precursors, which are secreted extracellularly where polymerisation into amyloid filaments follows. ${ }^{12}$ This hypothesis is supported by autoradiographic studies of isolated spleen explants and cultures. ${ }^{13}$

Amyloid is mostly located in connective tissue, that is, around blood vessels, in nodules, or between parenchymal cells. ${ }^{9}$ This type of distribution is seen in nerve, skeletal muscle and heart muscle. ${ }^{914}$ The oval masses as described in this study (figs $2,5,6$ ) may contribute significantly to the muscular hypertrophy and to the formation of nodules within the muscles. These nodules may also contribute to muscle weak

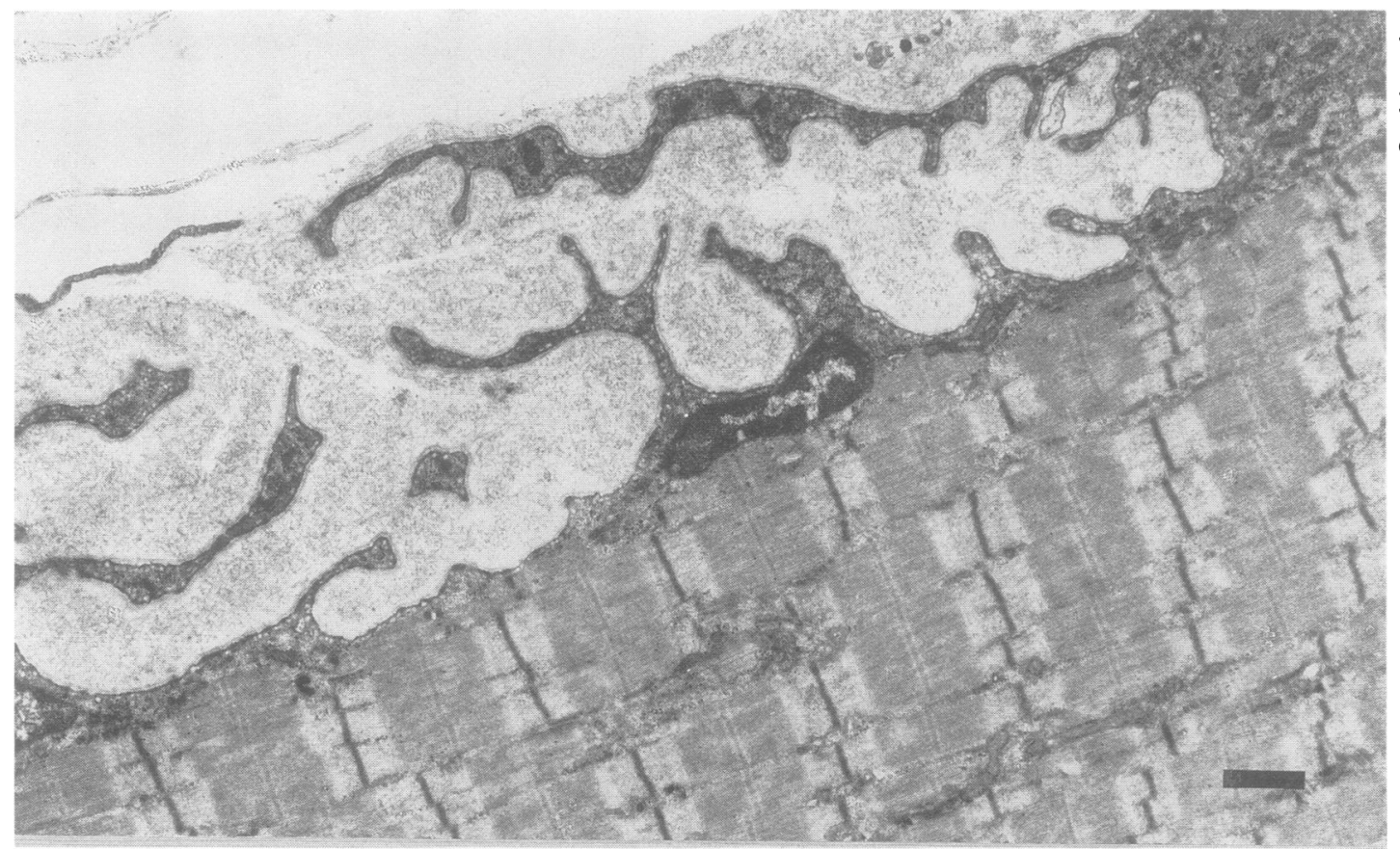

Fig 4 Electronmicrograph (case 2, left biceps brachii muscle). Elongated slender folds of the muscle fibre membrane extend into the amyloid layer covering the fibre. Bar: $1 \mu \mathrm{m}$. 

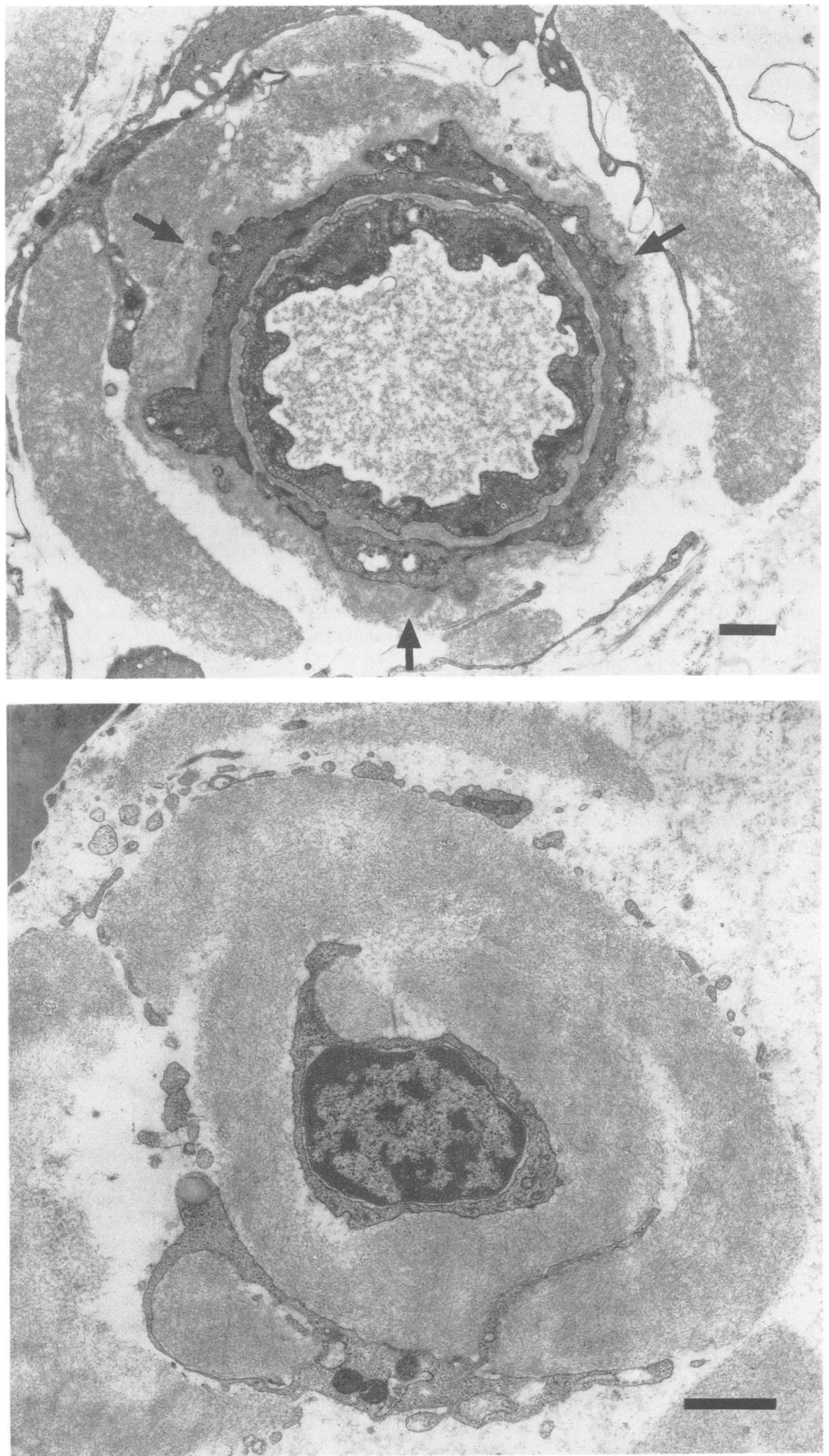

Fig 5 Electronmicrograph (same biopsy as fig 2). The basal lamina surrounding $a$ small vessel is covered by a shallow layer of amyloid (arrows). Oval masses of amyloid filaments are located nearby and are partly bounded or separated by fibroblasts. Bar: $1 \mu \mathrm{m}$.

Fig 6 Electronmicrograph (same biopsy as fig 2). A fibroblast is present in the centre of an oval mass of amyloid flaments. There are processes of other fibroblasts at the periphery of the mass. Bar: $1 \mu \mathrm{m}$. 
ness by compression of nearby muscle fibres. The amyloid layers upon the muscle fibres (figs 2, 3, 4) have been thought to interfere with normal propagation of action potentials along the sarcolemmal membrane. $^{7}$ The layers around the muscle fibres and those around the vessels (fig 5) might also interfere with the exchange of nutrients and waste products. In this regard, the excessive folding of some muscle fibre membranes (fig 4) is of interest and might be interpreted as attempt of muscle fibres to enlarge their exchange surfaces.

A study of amyloid deposition in cardiac musculature has revealed characteristic differences. In senile cardiac amyloidosis, amyloid is deposited mainly in global masses, whilst in plasma cell dyscrasia amyloidosis, it is deposited in a much more mixed pattern with both globules and layers upon muscle fibres and vessels. ${ }^{14}$

Amyloid depositions in skeletal musculature leading to myopathy has only been reported in a limited number of patients with cell plasma dyscrasia. $^{5-715-20}$ The most prominent findings are abnormal firmness and pseudohypertrophy of the musculature, and palpable tumours within muscles. Macroglossia to a degree that sometimes precludes closure of the mouth, dysphagia and hoarseness of the voice may also occur. Mobility may be hampered, and generalised or predominantly proximal muscle weakness may be present. Some patients have aching pain in muscles. In contrast with this syndrome our patients show that weakness and atrophy of proximal limb muscles may be the sole manifestations of amyloid myopathy. This form of amyloid myopathy is not entirely undocumented in the literature. Kyle and Greipp ${ }^{21}$ in their survey on 229 patients mention three patients with atrophy and progressive muscular weakness. In such patients, amyloid filaments may be deposited predominantly upon muscle fibres and around vessels with few if any oval masses.

Amyloid myopathy should be included in the differential diagnosis when proximal limb muscle weakness without pseudohypertrophy, develops in middle aged or elderly patients. In such cases a search for plasma cell dyscrasia should be made.

The authors are grateful to $\mathrm{Mr} \mathrm{H}$ Veldman for photography and helpful discussion, to Mrs A Jennekens-
Schinkel for critically reading the manuscript and to Mrs B Kraan and Mrs M Schipper for secretarial assistance.

\section{References}

1 Engel AG, Banker BQ, eds. Myology, New York: McGraw-Hill Book Co. 1986:1033.

2 Mastaglia FL, Walton J. Skeletal Muscle Pathology. Edinburgh: Churchill Livingston 1982.

3 Dubowitz VC. Muscle Biopsy. A practical approach. 2nd ed. London: Ballière Tindall 1985.

4 Carpenter S, Karpati G. Pathology of Skeletal Muscle. New York: Churchill Livingstone, 1984.

5 Martin JJ, Van Bogaert L, Van Damme L, Peremans E. Sur une pseudo-myopathie ligneuse generalisée par amyloidose primaire endomysio-vasculaire. J Neurol Sci 1970;11:147-66.

6 Whitaker JN, Hashimoto K, Quinones M. Skeletal muscle pseudohypertrophy. Neurology 1977;27:47-54.

7 Ringel SP, Claman HN. Amyloid associated muscle pseudohypertrophy. Arch Neurol 1982;39:413-7.

8 Pearse AEG. Histochemistry. Theoretical and Applied. Vol. II. 4th ed. Edinburgh: Churchill Livingstone 1985:578-81.

9 Cohen AS, Rubinow A. Amyloid polyneuropathy. In: Dyck PJ, Thomas PK, Lambert EH, Bunge R, eds. Peripheral Neuropathy Vol. II. 2nd ed. Philadelphia: WB Saunders 1984:1866-98.

10 Scully RE, Mark EJ, McNeely BK. Weekly clinicopathological exercises. Case 26-1986. $N$ Engl J Med 1986;315:45-55.

11 Dalakas MC, Cunningham G. Characterization of amyloid deposits in biopsies of 15 patients with "sporadic" (nonfamilial or plasma cell dyscrasic) amyloid polyneuropathy. Acta Neuropathol (Berl) 1986;69:66-72.

12 Ghadially FN. Ultrastructural Pathology of the Cell and Matrix. 2nd ed. London: Butterworths, 1982:920.

13 Cohen AS, Gross E, Shirahama T. The light and electron microscopic autoradiographic demonstration of local amyloid formation in spleen explants. Am J Pathol 1965;47:1079.

14 Smith TJ, Kyle RA. Clinical significance of histopathological patterns of cardiac amyloidosis. Mayo Clin Proc 1984;59:547-55.

15 Lubarsch $O$. Zur Kenntnis ungewöhnlicher Amyloidablagerungen. Virchows Arch (A) 1929;271: 205-28.

16 Mollow H, Lebell H. Zur Klinik der systematisierten Amyloidablagerungen. Wien Arch Int Med 1932;22: 205-28.

17 Fisher JH, Thomson RA. Primary amyloidosis. Can Med Assoc J 1958;78:264-6.

18 Lange RK. Primary amyloidosis of muscle. South Med J 1974:63:321-3.

19 Bruni J, Bilbao JM, Pritzker KPH. Myopathy associated with amyloid angiopathy. Can J Neurol Sci 1977;4:77.

$20 \mathrm{Li} \mathrm{K}$, Hirawa K, Nunomura S, Murozumi H. Systemic amyloid myopathy. Light miscroscopic and fine structural study of the skeletal muscles with histochemical and immunohistochemical study of amyloid. Acta Neuropathol 1984;64:114-21.

21 Kyle RA, Greipp PR. Amyloidosis (AL). Clinical and laboratory features in 229 cases. Mayo Clin Proc 1983;58:665-83. 